antihypertensive and corticosteroids. The immunosuppressant treatment used was: mycophenolate mofetil, azatioprine, cyclophosphamide, tacrolimus and rituximab. Considering that the time of follow-up of patients varies, each temporary space was analyzed according to the number of patients. Figure 1 shows that at 6 months, $38 \%$ of patients had an improvement $>50 \%$ and $7.7 \%$ of patients achieved remission. After one year of treatment, $42 \%$ of patients presented improvement $>50 \%$ and $17 \%$ achieved remission but in $23 \%$ of patients two changes of immunosuppressive treatment was needed. At 24 months, 50\% of patients improved $>50 \%$ and $25 \%$ achieved remission; in $13 \%$ of patients it was necessary to make another treatment change. Finally, after 24 months, it was observed that $50 \%$ of patients achieved remission, $33 \%$ of patients presented an improvement $>50 \%$ and only $17 \%$ presented renal failure, and it was necessary to make another treatment change. Globally, in $36 \%$ of patients 1 or more changes of immunosuppressive treatment were necessary to achieve improvement $>50 \%$ or remission.

Conclusions Patients who do not experience an improvement $>50 \%$ in a period of 6 months are more likely to improve if a change in immunosuppressive treatment is made. It is necessary to extend the series to reach conclusions with statistical value.

Funding Source(s): No funding

\section{DAMAGE ACCRUAL IN SWEDISH SYSTEMIC LUPUS ERYTHEMATOSUS: SECONDARY SJÖGRENS SYNDROME IS AMONG THE FACTORS ASSOCIATED WITH INCREASED RISK}

${ }^{1}$ Martina Frodlund*, ${ }^{2}$ Sarah Reid, 'Jonas Wetterö, 'Örjan Dahlström, 'Christopher Sjöwall, ${ }^{2}$ Dag Leonard. ${ }^{1}$ Linköping University; ${ }^{2}$ Uppsala university

10.1136/lupus-2019-Ism.264

Background Although the expected survival of patients with systemic lupus erythematosus (SLE) has improved during the last 50 years, accrual of damage remains a critical concern. Acquired damage is tightly linked to decreased quality-of-life and premature death, and could be due to raised disease activity, drug-related side-effects or co-morbidities.

Methods Accumulation of organ damage was studied in 543 well-characterized and from 1998 and onwards consecutively recruited prevalent/incident SLE cases meeting the 1982 American College of Rheumatology (ACR82) and/or the 2012 SLICC criteria. The SLICC/ACR damage index (SDI) was used to estimate damage. Disease variables were evaluated regarding association with damage accrual, and time to first damage. Detailed information on clinical and immunological features, as well as damage in each SDI domain, where at hands. Standardized mortality rate was calculated and causes of death recorded. Comparisons between groups were performed using Chi-square- or Mann-Whitney U-tests, p-values $<0.05$ were considered significant.

Results $59 \%$ of the patients had an SDI score $1 \%$ and $25 \%$ had extensive damage defined as SDI 3. Patients with presence of damage (SDI 1) were significantly older at disease onset, had longer SLE duration and fulfilled further classification criteria. Caucasian ethnicity was more common among cases with damage. Having ACR82-defined neurologic disorder, antiphospholipid syndrome (APS), any antiphospholipid antibody (positive IgG anti-2-GPI or a lupus anticoagulant test, separately) as well as concomitant comorbidities such as hypertension, hyperlipidemia, diabetes, depression and secondary Sjögrens syndrome were associated with presence of damage (SDI 1). In addition to factors associated with SDI 1, serositis, renal and hematological disorder as well as interstitial lung disease and positive IgG anti-cardiolipin were associated with extensive damage (SDI 3). Time to first damage was significantly shorter for males and for cases with a positive lupus anticoagulant test, whereas APS patients were borderline significant. Cases with malar rash and anti-La/SSB antibodies had significantly longer time to first damage. Malignancy was the most common cause of death.

Conclusions Despite that Swedish health care is tax-funded and offers universal access, a considerable number of patients are affected by irreversible damage over time. We confirm previous observations for several damage associations and

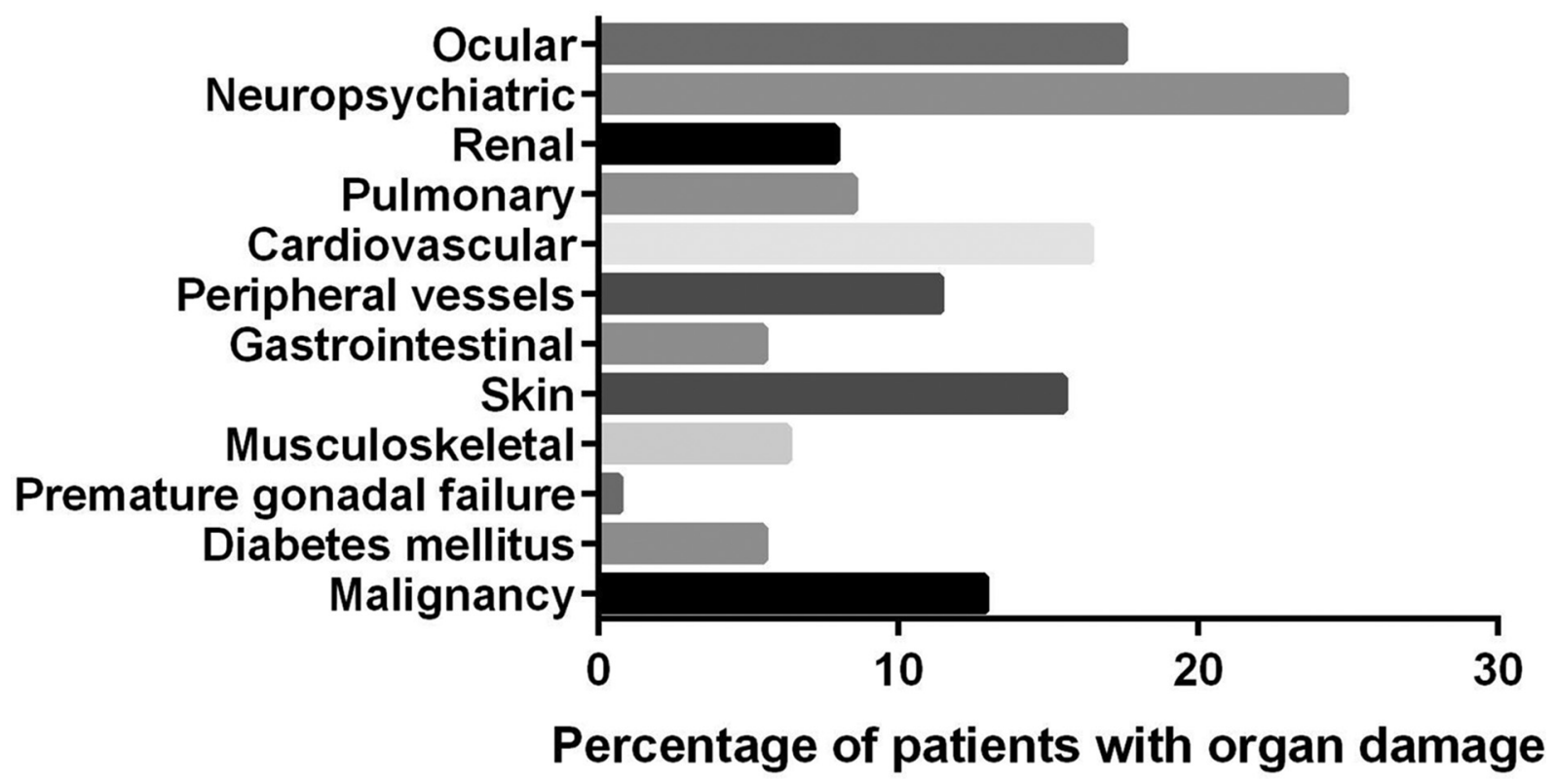

Abstract 264 Figure 1 Distribution of organ damage 
report secondary Sjögrens syndrome to be associated with an increased risk of organ damage in SLE.

Funding Source(s): This work was supported by grants from the Swedish Rheumatism Association, the County Council of Östergötland, the Swedish Society of Medicine, the King Gustaf Vs 80 year anniversary foundation and the King Gustaf V and Queen Victorias Freemasons foundation.

\section{ASSOCIATION OF PCSK9 SERUM LEVELS WITH LIPID METABOLISM DYSREGULATION, ACTIVITY/DAMAGE SCORES AND SUBCLINICAL ATHEROSCLEROSIS IN SLE PATIENTS}

${ }^{1}$ Ivan A Ferraz-Amaro*, ${ }^{1}$ Hiurma Sanchez, ${ }^{2}$ Juan Carlos Quevedo-Abeledo, ${ }^{3}$ ñigo RúaFigueroa, ${ }^{4}$ Beatriz Tejera-Segura, ${ }^{5}$ Antonia De Vera-Gonzalez, ${ }^{5}$ Alejandra González-Delgado, ${ }^{5}$ Laura De Armas-Rillo, ${ }^{5}$ Federico Díaz-González. ${ }^{1}$ Servicio de Reumatología; ${ }^{2}$ Hospital Dr. Negrin; ${ }^{3}$ Department of Rheumatology, Dr Negrín General University Hospital, Las Palmas de Gran Canaria; ${ }^{4}$ Hospital Insular; ${ }^{5}$ Hospital Universitario de Canarias

\subsection{6/lupus-2019-Ism.265}

Background SLE patients are characterized by a lipid metabolism dysregulation. Proprotein convertase subtilisin/kexin type 9(PCSK9) regulates cholesterol metabolism through low-density-lipoprotein (LDL) receptor degradation. PCSK9 has been linked to cardiovascular risk(CVR) in general population. The purpose of this study is to examine whether PCSK9 levels are related to disease activity, damage and severity scores; abnormalities in the lipid profile; and the subclinical atherosclerosis that occur in SLE patients.

Methods Cross-sectional study that encompasses 195 SLE patients. PCSK9 and lipoproteins serum concentrations were assessed. Activity (SLEDAI), severity (Katz) and damage (SLICC) index scores, and carotid ultrasound sonography were evaluated. A multivariable analysis was performed to evaluate the association of PCSK9 with SLE related dyslipidemia, subclinical atherosclerosis and activity/damage status

Results In the univariate analysis, body mass index, waist circumference, traditional CVR factors and triglycerides were related with PSCK9 serum levels. On the contrary, HDL cholesterol and apolipoprotein A levels showed a negative association. LDL cholesterol exhibited a trend to a negative association (beta coeff. $-0.30,95 \%$ CI $-0.67-0.07, p=0.11$ ). Carotid plaques and cIMT were not associated with PCSK9 levels although a trend was observed. Patients with longer disease duration (beta coeff. 1.25, 95\% CI 0.15-2.35, p=0.026) and higher $\mathrm{C}$ reactive protein levels (beta coeff. $1.42,95 \% \mathrm{CI}$ 0.61-2.22, $\mathrm{p}=0.00$ ) disclosed higher PCSK9 levels. Prednisone intake was positively associated with PCSK9 (beta coeff. $35.48,95 \%$ CI 14.29-56.6), $\mathrm{p}=0.001)$, and patients that were taking any DMARD or hydroxicloroquine disclosed significant lower levels of PCSK9 (beta coeff. -27.91, 95\% CI -54.5-$1.32, \mathrm{p}=0.040$ and beta coeff. $-39.21,95 \%$ CI $-62.21--$ 16.21, $\mathrm{p}=0.001$ respectively). Higher values of SLICC (beta coeff. 9.66, 95\% CI 4.47-14.84, p=0.000) and patients that were in the high/very high SLEDAI activity category (beta coeff. $62.9895 \%$ CI 18.10-107.86, $\mathrm{p}=0.006)$ disclosed significant higher values of PCSK9. When multivariate analysis was performed these positive associations with both SLICC and SLEDAI and the use of prednisone were maintained, as well as negative associations with LDL levels and the use of hydroxychloroquine.
Conclusions PCSK9 serum levels are independently related to SLE activity and damage scores. This would imply that the mechanisms leading to lipid metabolism dysregulation in SLE patients may be mediated or be a consequence of PCSK9.

Funding Source(s): This work was supported by a grant to I.FA. from the Spanish Ministry of Health, Subdirección General de Evaluación y Fomento de la Investigación, Plan Estatal de Investigación Científica y Técnica y de Innovación 2013-2016 and by Fondo Europeo de Desarrollo Regional - FEDER (Fondo de Investigaciones Sanitarias, FIS PI14/00394, PI17/ 00083)

\section{ANTI-OVARIAN ANTIBODIES ARE NOT FOUND MORE COMMONLY AMONG SLE WOMEN WITH PRE-MATURE MENOPAUSE}

${ }^{1}$ Martha Tsaliki, ${ }^{2}$ Kristi A Koelsch, ${ }^{3}$ Eliza Chakravarty, ${ }^{4} \mathrm{Hal}$ Scofield*. ${ }^{1}$ University of Oklahoam Health Sciences Center; ${ }^{2}$ Oklahoma Medical Research Foundation; Univesrity of Oklahoma Health Sciecnes Center; ${ }^{3}$ Oklahoma Medical Research Foundation; University of Oklahoma; ${ }^{4}$ Oklahoma Medical Research Foundation; University of Oklahoma; Oklahoma City US Department of Veterans Affairs Medical Center

\subsection{6/lupus-2019-Ism.266}

Background Systemic lupus erythematosus (SLE) is commonly treated with broad immunosuppression, including cyclophosphamide. Use of this agent can result in ovarian toxicity leading to premature menopause. Ovarian failure is associated with concomitant hypothyroidism. We hypothesized that hypothyroidism might be a marker for ovarian autoimmunity because organ-specific autoimmune diseases tend to occur together. We undertook this study to determine whether antiovarian antibodies are associated with premature ovarian failure among SLE women who received cyclophosphamide.

Methods All SLE women with a history of cyclophosphamide therapy were identified in the Lupus Registry and Repository (LFRR). All subjects were shown to meet the revised ACR classification criteria. Premature menopause was defined as spontaneous lack of menstrual periods prior to age 45. Antiovarian antibodies were measured by ELISA (Anti-Ovarian Ab ELISA, IBL, Minneapolis, catalog \# IB9184). Data were analyzed by Students $\mathrm{T}$ test and chi square testing. The protocol received ethical approval from the University of Oklahoma Health Sciences Center and Oklahoma Medical Research Foundation IRBs.

Results Among 3000 SLE women enrolled in the LFRR who had received cyclophosphamide, we found 169 with menopause before age 45 along with 73 who underwent menopause after age 45 and 16 patients over age 45 who were still having menstrual periods at the time of evaluation. Thus, there were a total of 89 SLE women who did not have premature menopause. Mean anti-ovarian antibodies in the 169 with premature ovarian failure was 16.2 units $(\mathrm{SD}=20.3)$, while the mean among those without premature menopause was 17.4 units $(\mathrm{SD}=21.7)$. These values were not statistically different between the groups. 11 of 169 (6.5\%) prematurely menopausal SLE women had a positive result and 8 of 89 $(8.9 \%)$ without premature menopausal were positive $(2=0.53$, $\mathrm{p}=0.46, \mathrm{OR}=1.02$ (95\% CI 0.95-1.1).

Conclusions Anti-ovarian antibodies were present at low levels ( $\sim 10 \%$ positivity) among women with SLE. However, the presence of these antibodies was not related to premature ovarian failure after cyclophosphamide. 